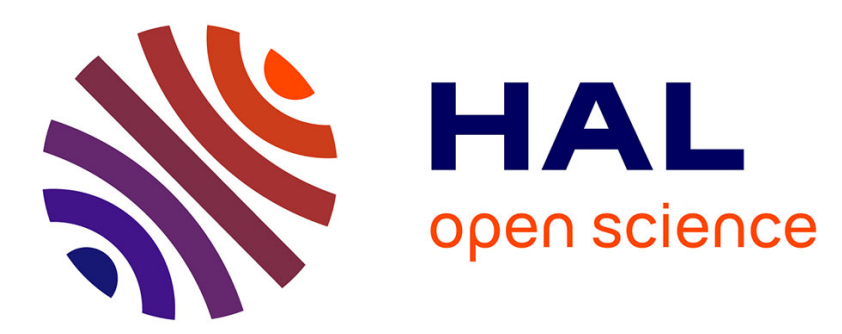

\title{
Mesure des temps de relaxation spin-réseau du rubis avec des structures maser en transmission \\ J.C. Jezequel
}

\section{To cite this version:}

J.C. Jezequel. Mesure des temps de relaxation spin-réseau du rubis avec des structures maser en transmission. Revue de Physique Appliquée, 1969, 4 (3), pp.341-343. 10.1051/rphysap:0196900403034100 . jpa-00243292

\section{HAL Id: jpa-00243292 https://hal.science/jpa-00243292}

Submitted on 1 Jan 1969

HAL is a multi-disciplinary open access archive for the deposit and dissemination of scientific research documents, whether they are published or not. The documents may come from teaching and research institutions in France or abroad, or from public or private research centers.
L'archive ouverte pluridisciplinaire HAL, est destinée au dépôt et à la diffusion de documents scientifiques de niveau recherche, publiés ou non, émanant des établissements d'enseignement et de recherche français ou étrangers, des laboratoires publics ou privés. 


\title{
REVUE DE PHYSIQUE APPLIQUÉE
}

\author{
Supplément au "Journal de Physique "
}

\section{MESURE DES TEMPS DE RELAXATION SPIN-RÉSEAU DU RUBIS AVEG DES STRUGTURES MASER EN TRANSMISSION}

\author{
Par J. G. JÉZÉQUEL, \\ G.N.E.T., Centre de Recherches de Lannion (22). \\ (Reçu le 23 janvier 1969.)
}

\begin{abstract}
Résumé. - On décrit un processus de mesure des temps de relaxation spin-réseau $T_{1}$ du rubis, dans des structures maser en transmission. L'élément de base du montage dérivé de celui de Bowers et Mims”est un pont constitué de deux tés magiques. Le dispositif a été utilisé avec un maser à une cavité en transmission et un maser à ondes progressives, la fréquence d'interrogation étant voisine de $4 \mathrm{GHz}$, la fréquence de saturation étant voisine de $4 \mathrm{GHz}$ ou de $30 \mathrm{GHz}$. Quelques résultats expérimentaux avec un maser à une cavité en transmission illustrent la méthode.
\end{abstract}

\begin{abstract}
We describe an apparatus for spin-lattice relaxation time measurements with ruby transmission type masers. The basic unit of the equipment derived from the one described by Bowers and Mims, is a microwave bridge with two magic tees. Measurements have been carried out with a two port cavity maser and a travelling wave maser. The observation of relaxation process is done at a frequency of about $4 \mathrm{GHz}$, the pump frequency being about $4 \mathrm{GHz}$ or $30 \mathrm{GHz}$. Some typical results for a two port cavity maser are given.
\end{abstract}

I. Introduction. - Nous avons appliqué la méthode de Bowers et Mims [1] à la mesure des temps de relaxation spin-réseau $T_{1}$ de rubis placés dans différentes structures maser en transmission mises au point dans notre laboratoire [2], [3].

La fréquence de fonctionnement des masers est de $4 \mathrm{GHz}$, celle de pompe étant de $30 \mathrm{GHz}$. Les rubis ont une concentration $c$ voisine de $0,035 \%$ (rapport du nombre d'ions chrome au nombre d'ions aluminium); le champ magnétique continu $\left(\mathbf{H}_{0}=3300 \mathrm{EE}\right)$ est perpendiculaire à l'axe optique du cristal. Aux basses températures et pour des concentrations faibles, on sait [1], [4] que la relaxation spin-réseau se traduit par la loi de variation suivante de la surtension paramagnétique $Q_{m}$ du cristal en fonction du temps $t$ :

$$
\frac{1}{Q_{m_{0}}}-\frac{1}{Q_{m}}=C \exp \left[-\frac{t}{T_{1}}\right]
$$

$Q_{m_{\mathrm{v}}}$ étant sa valeur à la fin de la relaxation, et $C$ une constante.

Nous allons montrer comment on peut déduire les variations de $\frac{1}{Q_{m}}$ en fonction du temps des variations des caractéristiques hyperfréquences (gain, absorption, niveau de sortie) des structures utilisées; le tracé de la relation (1) sur papier semi-logarithmique nous permet alors de déterminer $T_{1}$.

II. Cas du maser à une cavité en transmission. La structure hyperfréquence a été décrite en [3]. Les pertes intrinsèques étant négligeables, nous aurons [5] les expressions suivantes pour le gain ou l'absorption, $Q_{e}$ désignant la surtension de couplage de la cavité :

$$
\begin{aligned}
\text { Gain } & =\left[1+\frac{Q_{e}}{Q_{m}}\right]^{-2} & \text { si } & Q_{m}<0 \\
\text { Absorption } & =\left[1+\frac{Q_{e}}{Q_{m}}\right]^{2} & \text { si } & Q_{m}>0 .
\end{aligned}
$$

Le montage expérimental utilisé lorsque la saturation en impulsions est faite à la fréquence de $30 \mathrm{GHz}$ est représenté sur la figure $1 \mathrm{a}$. Les puissances de sortie du maser (élément 6 de la figure $1 \mathrm{a}$ ) à une fréquence de l'ordre de $4 \mathrm{GHz}$ sont $P_{1}, P_{2}, P_{3}, P_{4}$ et $P_{5}$ (voir fig. 2). Il est possible, pour un signal quelconque à la sortie du maser, d'obtenir par la combinaison atténuateurdéphaseur de l'autre branche du pont (4 et 5) un signal résultant nul sur le mélangeur 11 . Nous effectuons le zéro du pont lorsque le champ magnétique continu $\mathbf{H}_{\mathbf{0}}$ est appliqué seul. 

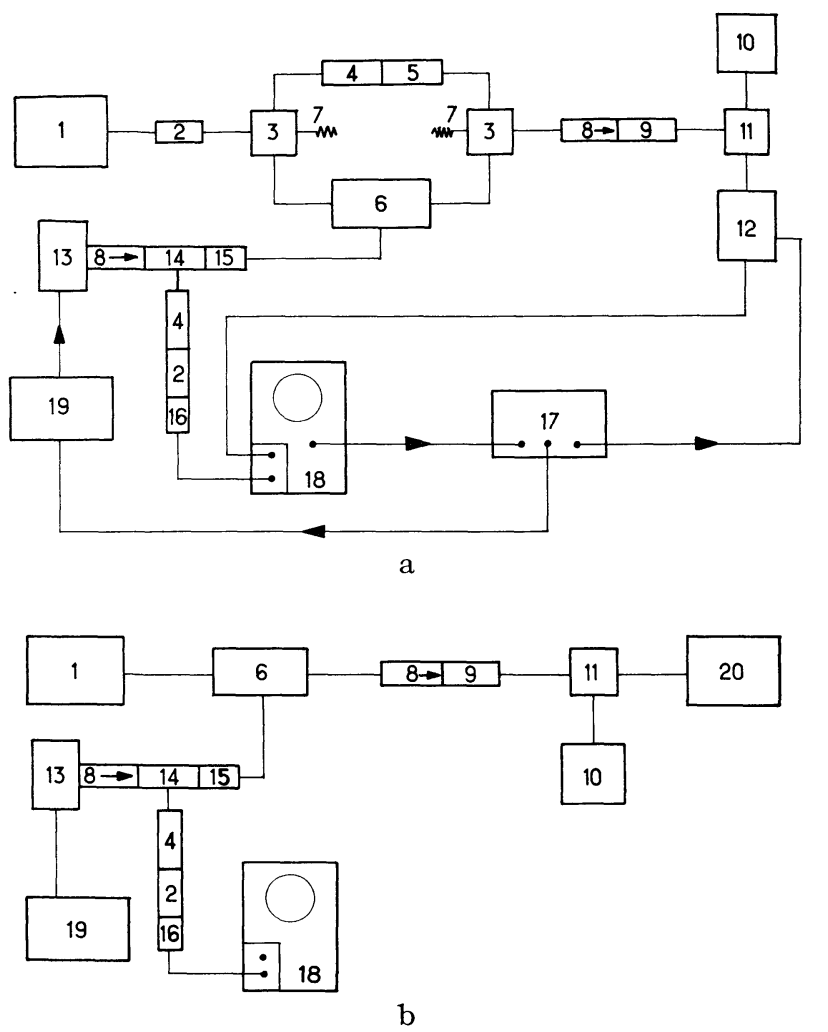

FIG. 1. - 1, Générateur d'interrogation avec atténuateur incorporé ; 2 , Ondemètre ; 3 , Té magique; 4 , Atténuateur ; 5, Déphaseur ; 6 , Structure maser ; 7 , Charge adaptée ; 8, Isolateur; 9, Atténuateur de précision ; 10, Oscillateur local ; 11, Mélangeur; 12, Amplificateur à fréquence intermédiaire (avec dispositif de blocage lors de la saturation) ; 13, Klystron de saturation ; 14, Coupleur $10 \mathrm{~dB} ; 15$, Atténuateur calibré ; 16, Détecteur ; 17, Générateur d'impulsions ; 18, Oscilloscope avec tiroir double trace; 19, Alimentation pour klystron; 20, Récepteur à fréquence intermédiaire.

1 a) Montage expérimental avec saturation et interrogation à des fréquences différentes.

$1 \mathrm{~b})$ Montage de mesure de l'absorption $A_{1}$ et du gain $G_{1}$.

Posons :

$$
\begin{gathered}
p_{m}=P_{m}-P_{2} \quad(m=1,2,3,4,5) \\
G_{1}=10 \log \frac{P_{5}}{P_{2}} ; \quad A_{1}=10 \log \frac{P_{1}}{P_{2}} \\
n=10 \log \frac{p_{5}}{p_{4}} .
\end{gathered}
$$

A partir des relations (2) et (3), on obtient alors : $\frac{Q_{e}}{Q_{m}}=\left[10^{-\frac{A_{1}}{10}}+10^{-\frac{n}{10}}\left(10^{\frac{G_{1}-A_{1}}{10}}-10^{\left.-\frac{A_{1}}{10}\right)}\right]^{-1 / 2}-1\right.$.
La méthode expérimentale est la suivante :

a) Le montage de la figure $1 \mathrm{~b}$ permet de mesurer l'absorption maximale $A_{1}$ à la fréquence de résonance de la cavité (champ $\mathbf{H}_{0}$ appliqué). L'atténuateur 9 nous donne le rapport entre les puissances transmises avec ou sans champ $\mathbf{H}_{0}$, la puissance de pompe n'étunt pas appliquée.

b) Nous réalisons alors le montage de la figure 1 a. On applique le champ $\mathbf{H}_{0}$, sans puissance de pompe, et on fait le zéro du pont $\left(p_{2}=0\right)$.

c) Des impulsions de puissance pompe (durée $\tau$ ) sont appliquées. On enregistre les courbes de relaxation (voir fig. 2), la graduation en décibels de l'atténuateur 9 de la figure 1 a étant sur zéro.

d) Toujours avec le montage de la figure $1 \mathrm{a}$, le klystron de pompe fonctionnant maintenant en ondes entretenues, on s'arrange pour obtenir un gain quelconque $G_{1}$ (niveau $p_{5}$ de la figure 2 ).

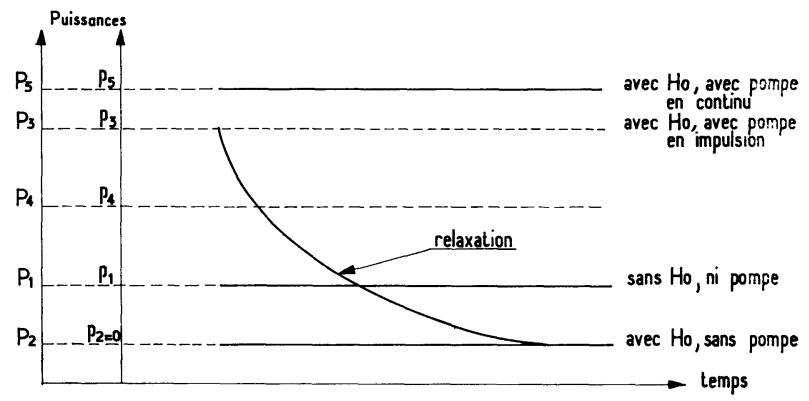

FIG. 2. - Puissances de sortie à $4 \mathrm{GHz}$ (avec saturation à $30 \mathrm{GHz}): \mathbf{H}_{0}$, champ magnétique continu ; $P_{1}, P_{2}$, $P_{3}, P_{4}, P_{5}$ sont les puissances à la sortie du maser (élément 6 de la figure 1 a)) dans les cas suivants : $P_{1}, \mathbf{H}_{0}$ et pompe non appliqués; $P_{2}, \mathbf{H}_{0}$ appliqué, pompe non appliquée; $P_{3}, \mathbf{H}_{\mathbf{0}}$ appliqué, impulsion de pompe appliquée; $P_{4}$, en cours de relaxation; $P_{5}$, $\mathbf{H}_{0}$ appliqué, pompe appliquée en ondes entretenues; $p_{1}, p_{2}, p_{3}, p_{4}, p_{5}$, sont définis par les relations (3).

En affichant différentes atténuations $n=n_{1}, n_{2}, n_{3} \ldots$ sur l'atténuateur 9 , on obtient sur l'oscilloscope un ensemble de droites parallèles à l'axe horizontal (le niveau $p_{5}$ initial est atténué de $n$ décibels).

e) Nous reprenons alors le montage de la figure $1 \mathrm{~b}$ pour mesurer le gain $G_{1}$. L'atténuateur 9 nous permettra d'obtenir le rapport entre les puissances de sortie à $4 \mathrm{GHz}$ du maser, la puissance pompe étant soit appliquée comme en $d$ ), soit atténuée au maximum. f) Par la suite, la superposition des courbes des photographies de l'écran de l'oscilloscope obtenues en $c$ ) et $d$ ) (avec agrandissement sur un projecteur de profil) donnera, aux points d'intersection, plusieurs valeurs de $\frac{Q_{e}}{Q_{m}}$ en fonction du temps (voir relation (4)), la valeur de $\frac{Q_{e}}{Q_{m_{0}}}$
se déduisant de (4) pour $n$ infini. 
Dans le cas où la saturation et l'interrogation se font à la même fréquence, soit $4 \mathrm{GHz}$ dans notre cas, le montage de la figure $1 \mathrm{~b}$ est encore valable; celui de la figure 1 a est légèrement modifié : la sortie du klystron 13 est ramenée à l'entrée du maser par un coupleur. Les relations (3) et la figure 2 sont encore valables à condition de prendre $P_{5}$ identique à $P_{1}$ (ce qui entraîne $G_{1} \# A_{1}$ et $p_{5} \# p_{1}$ ).

On montre aisément que l'expression (4) reste encore valable $\left(G_{1}-A_{1}=0\right)$.

Expérimentalement, la seule différence avec le cas précédent réside dans le fait que le réseau de droites du paragraphe $d$ ) est obtenu avec champ magnétique $H_{0}$ et puissance pompe supprimés.

III. Cas du maser à ondes progressives. - De la même manière qu'au $\S \mathrm{II}$, on obtient [2], [5], la formule :

$C^{\prime}\left(\frac{1}{Q_{m_{0}}}-\frac{1}{Q_{m}}\right)=10 \log \left[1+10^{-\frac{n}{10}}\left(10^{\frac{G_{1}}{10}}-1\right)\right]$

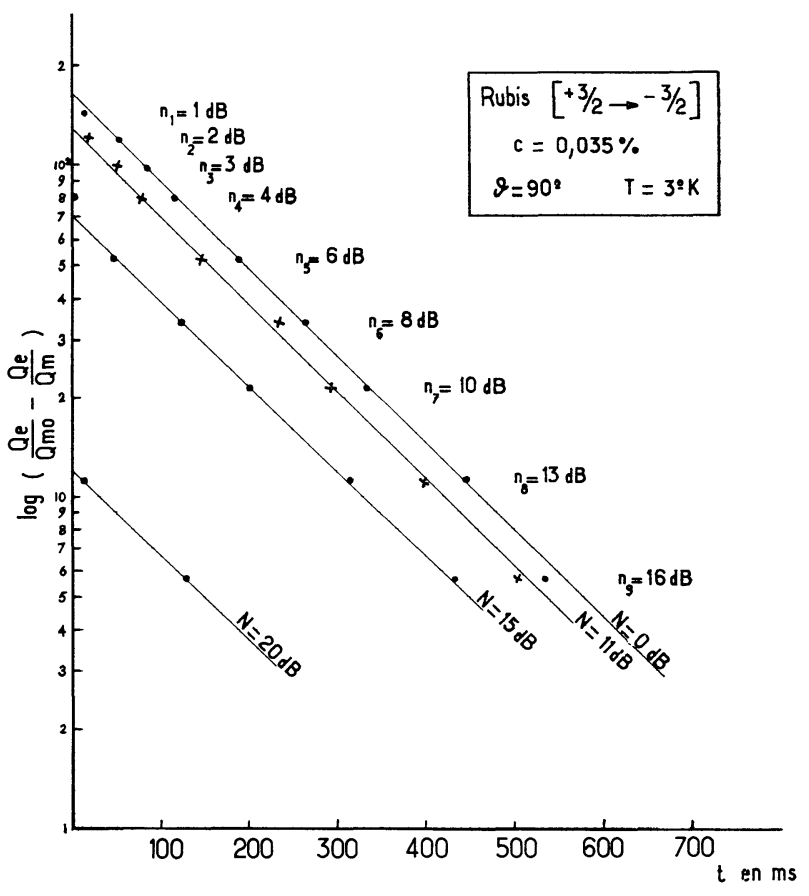

FIG. 3. - Courbe de relaxation typique pour une durée $\tau=1 \mathrm{~ms}$ de l'impulsion de saturation et pour différentes indications $N$ de l'atténuateur 15 de la figure 1 a (pour $N=0 \mathrm{~dB}$ la puissance initiale de saturation est environ $100 \mathrm{~mW}$ ).

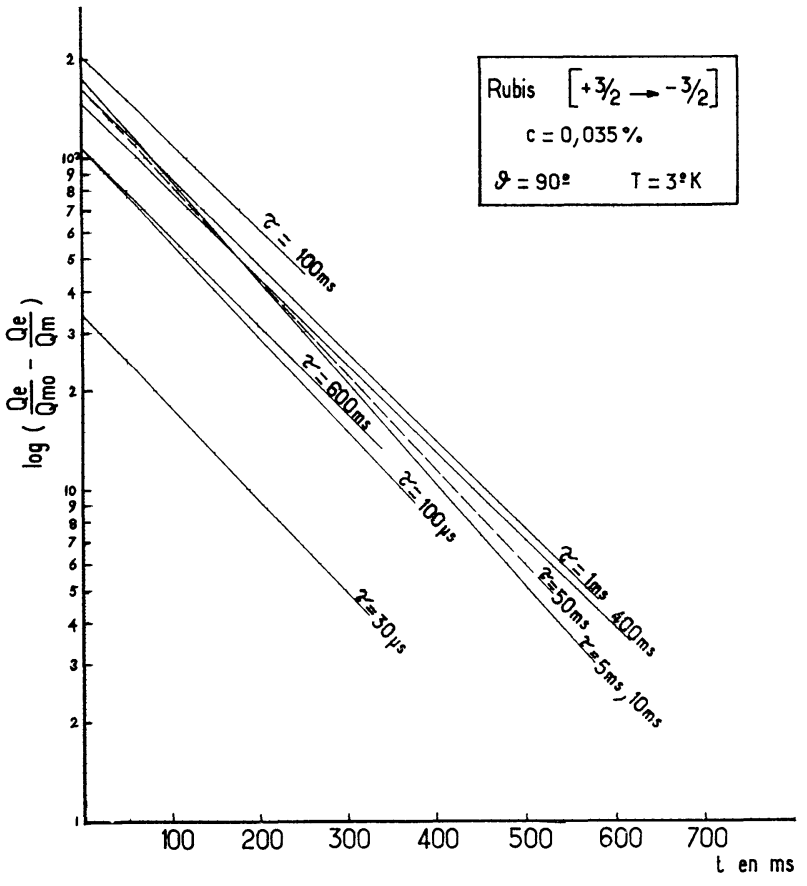

FIG. 4. - Courbes de relaxation pour différentes durées $\tau$ de l'impulsion de saturation.

$C^{\prime}$ étant une constante caractérisant la ligne en peigne utilisée; la méthode expérimentale est la même qu'au $\S$ II.

IV. Résultats expérimentaux. - Afin d'illustrer la méthode, nous donnons quelques résultats obtenus avec une structure à une cavité en transmission. Les résultats des figures 3 et $4-$ avec interrogation et saturation à $4002 \mathrm{MHz}$ - montrent qu'un seul temps $T_{1}$ est mis en évidence; $T_{1}$ est indépendant de la puissance et de la durée de l'impulsion de saturation. D'après la figure 4 , la valeur moyenne de $T_{1}$ est de $157 \mathrm{~ms}(+2 \%,-10 \%)$.

V. Conclusion. - La méthode décrite utilisant un pont - dont le zéro peut être vérifié très rapidement en cours de manipulation - est très pratique lorsque l'on dispose de structures en transmission ayant des largeurs de bande de plusieurs mégahertz (un générateur d'interrogation stabilisé ou un contrôle automatique de fréquence de l'oscillateur local 10 de la figure 1 a ne sont pas indispensables). Elle s'avère très intéressante pour l'étude des rubis placés dans les masers à ondes progressives. Les quelques résultats expérimentaux du chapitre IV sont en bon accord avec ceux qui sont généralement donnés pour le rubis.

\section{BIBLIOGRAPHIE}

[1] Bowers (K. D.) et Mims (W. B.), Phys. Rev., 1959, 115, 285-295.

[2] Nizery (A.) et Loriou (B.), Onde électrique, 1967, 121-128.
[3] JÉzÉQué, (J. C.), Rev. Phys. Appl., 1967, 2, 20-22.

[4] Donoho (P. L.), Phys. Rev., 1964, 133 A, 1080-1084.

[5] Siegman (A. E.), Microwave Solid State Masers, McGraw-Hill, New York, 1964. 